TRANSACTIONS OF THE

AMERICAN MATHEMATICAL SOCIETY

Volume 348, Number 1, January 1996

\title{
POLYNOMIAL STRUCTURES FOR NILPOTENT GROUPS
}

\author{
KAREL DEKIMPE, PAUL IGODT, AND KYUNG BAI LEE
}

\begin{abstract}
If a polycyclic-by-finite rank- $K$ group $\Gamma$ admits a faithful affine representation making it acting on $\mathbb{R}^{K}$ properly discontinuously and with compact quotient, we say that $\Gamma$ admits an affine structure. In 1977, John Milnor questioned the existence of affine structures for such groups $\Gamma$. Very recently examples have been obtained showing that, even for torsion-free, finitely generated nilpotent groups $N$, affine structures do not always exist. It looks natural to consider affine structures as examples of polynomial structures of degree one. We introduce the concept of a canonical type polynomial structure for polycyclic-by-finite groups. Using the algebraic framework of the Seifert Fiber Space construction and a nice cohomology vanishing theorem, we prove the existence and uniqueness (up to conjugation) of canonical type polynomial structures for virtually finitely generated nilpotent groups. Applying this uniqueness to a result going back to $\mathrm{Mal}^{\prime} \mathrm{cev}$, it follows that, for torsion-free, finitely generated nilpotent groups, each canonical polynomial structure is expressed in polynomials of limited degree. The minimal degree needed for obtaining a polynomial structure will determine the "affine defect number". We prove that the known counterexamples to Milnor's question have the smallest possible affine defect, i.e. affine defect number equal to one.
\end{abstract}

\section{InTRODUCTION AND MAIN RESULT}

Let $G$ be a connected and simply connected solvable Lie group. Milnor ([Mil77]) conjectured that $G$ admits a complete affinely flat structure invariant under left translations. Since then, a few papers claiming this conjecture affirmatively have been published, for example, ([Boy89], [Boy90] and [Nis90]).

With this in mind, the idea of a canonical type affine representation (implicitly born in [Sch74] and [Lee83]) for (virtually) torsion-free, finitely generated nilpotent groups and the iteration problem related to this have been investigated ([DI93]). Uniqueness of these representations was studied in ([IL90]).

However, today, counterexamples produced by Benoist ([Ben92]) and Burde and Grunewald ([BG93]) show that certain nilmanifolds do not admit a complete affinely flat structure. Consequently, it follows that Milnor's conjecture is false even in the nilpotent case.

Note that one can look at such a complete affinely flat structure as a "polynomial structure of degree 1". In a situation where "polynomial structures of degree 1" fail to exist, the next best structure will be "polynomial structure of higher degree".

Received by the editors May 8, 1994 .

1991 Mathematics Subject Classification. Primary 57S30, 22E40, 22E25 .

Key words and phrases. Lattices in nilpotent Lie groups, complete affinely flat structures.

The first author is Research Assistant of the National Fund For Scientific Research (Belgium).

(C)1996 American Mathematical Society 
The purpose of this paper is to prove that every torsion-free, finitely generated nilpotent group of rank $K$ admits a faithful "canonical type" representation into the group of polynomial diffeomorphisms of $\mathbb{R}^{K}$. This result will be achieved using the algebraic framework of the so-called Seifert Fiber Space construction. As a consequence of this approach, uniqueness of such canonical representations is also proved; moreover, existence and uniqueness stay valid for groups which are virtually nilpotent.

Based on the uniqueness and a well-known canonical polynomial structure found already by $\mathrm{Mal}^{\prime} \mathrm{cev}$, it looks interesting to introduce the notion of affine defect number for a torsion-free f.g. nilpotent group. Groups of affine defect 0 are exactly the fundamental groups of the complete affinely flat manifolds. Groups of affine defect $>0$ are those obstructing Milnor's question. In the closing section we prove that the examples produced by Benoist, Burde and Grunewald are of affine defect 1. More precisely, the 11-dimensional nilpotent Lie groups which fail to admit a complete affinely flat structure do admit a polynomial structure of degree 2 . This is the lowest possible such a structure.

\section{Canonical type structures}

It is well known ([Seg83, Lemma 6, p. 16]) that, if $\Gamma$ is a polycyclic-by-finite group, then there exists an ascending sequence (or filtration) of normal subgroups $\Gamma_{i}(0 \leq i \leq c+1)$ of $\Gamma$

$$
\Gamma_{*}: \Gamma_{0}=1 \subset \Gamma_{1} \subset \Gamma_{2} \subset \cdots \subset \Gamma_{c-1} \subset \Gamma_{c} \subset \Gamma_{c+1}=\Gamma
$$

for which

$$
\begin{gathered}
\Gamma_{i} / \Gamma_{i-1} \cong \mathbb{Z}^{k_{i}} \text { for } 1 \leq i \leq c \text { and some } k_{i} \in \mathbb{N}_{0} \text { and } \\
\Gamma / \Gamma_{c} \text { is finite. }
\end{gathered}
$$

Moreover, each $\Gamma_{i}$ can be chosen to be a characteristic subgroup of $\Gamma$. Let us call such a filtration of $\Gamma$ a torsion-free filtration. We use $K$ to denote the Hirsch number (or rank) of $\Gamma$. Often, we will also use $K_{i}=k_{i}+k_{i+1}+\cdots+k_{c}$ and $K_{c+1}=0$. It follows that $K=K_{1}$.

Definition 2.1. Assume $\Gamma$ is polycyclic-by-finite with a torsion-free filtration $\Gamma_{*}$. A set of generators $A=\left\{a_{1,1}, a_{1,2}, \ldots, a_{1, k_{1}}, a_{2,1}, \ldots, a_{c, k_{c}}, \alpha_{1}, \ldots, \alpha_{r}\right\}$ of $\Gamma$ will be called compatible with $\Gamma_{*}$ iff

$$
\forall i \in\{1,2, \ldots, c\}: a_{1,1}, a_{1,2}, \ldots, a_{i, k_{i}} \text { generates } \Gamma_{i} .
$$

It is clear at once that any torsion-free filtration $\Gamma_{*}$ of $\Gamma$ admits a compatible set of generators.

In the sequel, frequently we will work with quotient groups of $\Gamma$. In order to avoid complicated notations, we will write the same symbols for elements in $\Gamma$, as for their coset classes in a quotient group. E.g. we say that $\Gamma_{2} / \Gamma_{1}$ is the free abelian group generated by $a_{2,1}, a_{2,2}, \ldots, a_{2, k_{2}}$.

Now we introduce quickly the basic building blocks for the representations we are interested in (see [Lee83] and [CR77]):

$$
\begin{gathered}
\mathcal{M}\left(\mathbb{R}^{K}, \mathbb{R}^{k}\right)=\left\{\text { continuous maps } \lambda: \mathbb{R}^{K} \rightarrow \mathbb{R}^{k}\right\}, \\
\mathcal{H}\left(\mathbb{R}^{K}\right)=\left\{\text { homeomorphisms } h: \mathbb{R}^{K} \rightarrow \mathbb{R}^{K}\right\} .
\end{gathered}
$$


$\mathcal{M}\left(\mathbb{R}^{K}, \mathbb{R}^{k}\right)$ is an abelian group (for the addition of maps) and can be made into a $\operatorname{GL}(k, \mathbb{R}) \times \mathcal{H}\left(\mathbb{R}^{K}\right)$-module, if we define

$$
{ }^{(g, h)} \lambda=g \circ \lambda \circ h^{-1}, \quad \forall \lambda \in \mathcal{M}\left(\mathbb{R}^{K}, \mathbb{R}^{k}\right), \forall g \in \mathrm{GL}(k, \mathbb{R}), \forall h \in \mathcal{H}\left(\mathbb{R}^{K}\right) .
$$

We remark that there is an embedding $\mathcal{M}\left(\mathbb{R}^{K}, \mathbb{R}^{k}\right) \rtimes\left(\mathrm{GL}(k, \mathbb{R}) \times \mathcal{H}\left(\mathbb{R}^{K}\right)\right) \hookrightarrow$ $\mathcal{H}\left(\mathbb{R}^{k+K}\right)$ with

$$
\begin{gathered}
(\lambda, g, h)(x, y)=(g(x)+\lambda h(y), h(y)), \\
\forall x \in \mathbb{R}^{k}, \forall y \in \mathbb{R}^{K}, \forall \lambda \in \mathcal{M}\left(\mathbb{R}^{K}, \mathbb{R}^{k}\right), \forall g \in \mathrm{GL}(k, \mathbb{R}), \forall h \in \mathcal{H}\left(\mathbb{R}^{K}\right) .
\end{gathered}
$$

Definition 2.2. Assume $\Gamma$ is polycyclic-by-finite with a torsion-free filtration $\Gamma_{*}$. A representation $\rho=\rho_{0}: \Gamma \rightarrow \mathcal{H}\left(\mathbb{R}^{K}\right)$ will be called of canonical type with respect to $\Gamma_{*}$ iff it induces a sequence of representations:

$$
\rho_{i}: \Gamma / \Gamma_{i} \rightarrow \mathcal{H}\left(\mathbb{R}^{K_{i+1}}\right), \quad(1 \leq i \leq c)
$$

such that for all $i$ the following diagram commutes:

$$
\begin{aligned}
& 1 \rightarrow \mathbb{Z}^{k_{i}} \cong \Gamma_{i} / \Gamma_{i-1} \rightarrow \Gamma / \Gamma_{i-1} \quad \rightarrow \quad \Gamma / \Gamma_{i} \quad \rightarrow 1 \\
& \downarrow j \quad \downarrow \rho_{i-1} \quad \downarrow B_{i} \times \rho_{i} \\
& 1 \rightarrow \mathcal{M}\left(\mathbb{R}^{K_{i+1}}, \mathbb{R}^{k_{i}}\right) \rightarrow \mathcal{M} \rtimes(G \times \mathcal{H}) \rightarrow \mathrm{GL}\left(k_{i}, \mathbb{Z}\right) \times \mathcal{H}\left(\mathbb{R}^{K_{i+1}}\right) \rightarrow 1
\end{aligned}
$$

where

- $\mathcal{M} \rtimes(G \times \mathcal{H})$ stands for $\mathcal{M}\left(\mathbb{R}^{K_{i+1}}, \mathbb{R}^{k_{i}}\right) \rtimes\left(\mathrm{GL}\left(k_{i}, \mathbb{Z}\right) \times \mathcal{H}\left(\mathbb{R}^{K_{i+1}}\right)\right)$,

- $j(z): \mathbb{R}^{K_{i+1}} \rightarrow \mathbb{R}^{k_{i}}: x \mapsto z, \forall z \in \mathbb{Z}^{k_{i}}$ and

- $B_{i}: \Gamma / \Gamma_{i} \rightarrow \mathrm{GL}\left(k_{i}, \mathbb{Z}\right)$ denotes the action of $\Gamma / \Gamma_{i}$ induced on $\mathbb{Z}^{k_{i}}$ by conjugation in $\Gamma / \Gamma_{i-1}$.

Let us establish the following results, which will be of use in a characterisation of canonical type representations.

Theorem 2.3. Let $\Gamma$ be any group acting on a contractible space $\widetilde{M}$ and containing a normal subgroup $\Gamma^{\prime}$ of finite index, which acts freely and properly discontinuously on $\widetilde{M}$, such that the quotient space $M=\Gamma^{\prime} \backslash \widetilde{M}$ is a closed aspherical manifold. Then, the kernel of the induced action of $F=\Gamma / \Gamma^{\prime}$ on $M$ is the isomorphic image of the torsion of $C_{\Gamma}\left(\Gamma^{\prime}\right)$ inside $F$. In fact, the set of all torsion of $C_{\Gamma}\left(\Gamma^{\prime}\right)$ forms a characteristic subgroup of $\Gamma$, and equals the kernel of the action of $\Gamma$ on $\widetilde{M}$.

Proof. Let $t$ be a torsion element in $C_{\Gamma}\left(\Gamma^{\prime}\right)$. This means that in the commutative diagram

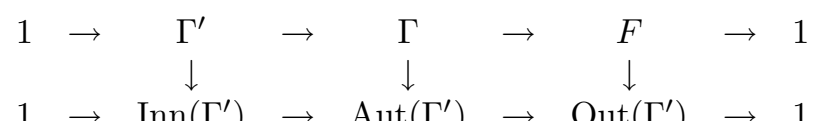

the group $T$ generated by $t$ maps trivially into $\operatorname{Aut}\left(\Gamma^{\prime}\right)$. (The vertical maps are induced by conjugation in $\Gamma$ ). By [LR81, Lemma 1] the image of $T$ in $F$ acts trivially on $M$. This image is isomorphic to $T$, as $\Gamma^{\prime}$ is torsion-free. Conversely, if $T^{\prime}$ is a subgroup of $F$ acting trivially on $M$, then $T^{\prime}$ is the isomorphic image of a subgroup $T$ of $\Gamma$ (this lifting of $T^{\prime}$ back to $\Gamma$ can be done already if the $T^{\prime}$ action on $M$ has a fixed point). Clearly, then $T$ commutes with $\Gamma^{\prime}$ so that $T \subset C_{\Gamma}\left(\Gamma^{\prime}\right)$.

For the last statement, note that a torsion element of $\Gamma$ acts effectively on $\widetilde{M}$ if and only if the corresponding element of $F$ acts effectively on $M$. 
As an immediate consequence, we find the following reformulation of the above theorem in the situation we're interested in.

Corollary 2.4. Let $\Gamma$ be a polycyclic-by-finite group, with Hirsch number $K$. Let $N$ be the $\Gamma_{c}$ in a torsion-free filtration of $\Gamma$, and let $F$ denote the set of torsion of $C_{\Gamma}(N)$. For any properly discontinuous action of $\rho: \Gamma \rightarrow \mathbb{R}^{K}, F$ is the kernel of $\rho$. Moreover, $F$ is the maximal finite normal subgroup of $\Gamma$.

We are now ready to summarize some important results on (general) representations of canonical type. For elements $x \in \mathbb{R}^{K}$ we will label the coordinates as follows:

$$
x=\left(x_{1,1}, x_{2,1}, \ldots, x_{1, k_{1}}, x_{2,1}, \ldots, x_{2, k_{2}}, x_{3,1}, \ldots, x_{c, k_{c}}\right) .
$$

We also write $x_{i}$ to indicate all variables $\left(x_{i, 1}, x_{i, 2}, \ldots, x_{i, k_{i}}\right)$. If $\rho=\rho_{0}: \Gamma \rightarrow$ $\mathcal{H}\left(\mathbb{R}^{K}\right)$ is a representation, then for each $\gamma \in \Gamma$ there exist continuous functions $h_{i, j}^{\gamma}: \mathbb{R}^{K} \rightarrow \mathbb{R}$ such that

$$
\rho(\gamma): \mathbb{R}^{K} \rightarrow \mathbb{R}^{K}: x \mapsto\left(h_{1,1}^{\gamma}(x), h_{1,2}^{\gamma}(x), \ldots, h_{c, k_{c}}^{\gamma}(x)\right) .
$$

In a way similar as for the $x_{i, j}$ we use $h_{i}^{\gamma}$ to indicate $\left(h_{i, 1}^{\gamma}(x), h_{i, 2}^{\gamma}(x), \ldots, h_{i, k_{i}}^{\gamma}(x)\right)$.

Theorem 2.5. Let $\Gamma$ be polycyclic-by-finite, $\Gamma_{*}$ be a torsion-free filtration of $\Gamma$. Then, there exists a representation $\rho: \Gamma \rightarrow \mathcal{H}\left(\mathbb{R}^{K}\right)$ which is of canonical type with respect to $\Gamma_{*}$. Moreover, any two such representations $\rho, \rho^{\prime}: \Gamma \rightarrow \mathcal{H}\left(\mathbb{R}^{K}\right)$ are conjugate to each other inside $\mathcal{H}\left(\mathbb{R}^{K}\right)$. Also, for each canonical type representation $\rho: \Gamma \rightarrow \mathcal{H}\left(\mathbb{R}^{K}\right)$ we have:

(1) $\Gamma$ acts properly discontinuously on $\mathbb{R}^{K}$, via $\rho$ and with compact quotient.

(2) $\operatorname{ker} \rho=$ the maximal finite normal subgroup of $\Gamma$, so $\rho$ is effective iff $\Gamma$ is almost torsion-free (i.e. has no non-trivial finite normal subgroups) (see [DI94]).

(3) $\forall \gamma \in \Gamma:$

$$
\begin{aligned}
\rho(\gamma): \mathbb{R}^{K} \rightarrow \mathbb{R}^{K}: x & \mapsto\left(h_{1}^{\gamma}(x), h_{2}^{\gamma}(x), \ldots, h_{c}^{\gamma}(x)\right) \text { is such that } \\
h_{i}^{\gamma}(x) & =B_{i}(\gamma) x_{i}+g_{i}^{\gamma}\left(x_{i+1}, x_{i+2}, \ldots, x_{c}\right),
\end{aligned}
$$

for some continuous function $g_{i}^{\gamma}: \mathbb{R}^{K_{i+1}} \rightarrow \mathbb{R}^{k_{i}}$.

(4) $\Gamma_{i}$ acts trivially on $\mathbb{R}^{K_{i+1}}$ and via affine transformations on $\mathbb{R}^{k_{i}}$.

Proof. The proof of the existence and uniqueness of a canonical type representation can be found in [LR84]. The reader may also consult [LR84] to see that the action of $\Gamma$ defined on $\mathbb{R}^{K}$ is properly discontinuous and that the orbit space is compact.

(2) follows from Corollary 2.4.

(3) and (4) are immediate consequences of the iterative way of building up $\rho$ (see also (1)).

Having this concept of a (general) canonical type representation, it is clear now that to get a nicer geometric structure one needs to use smaller subgroups of $\mathcal{H}\left(\mathbb{R}^{K}\right)$.

In view of the iterative set up, it is however necessary that these subgroups satisfy one crucial condition: assume we restrict $\mathcal{M}\left(\mathbb{R}^{K}, \mathbb{R}^{k}\right)$ to a subspace $\mathcal{S}\left(\mathbb{R}^{K}, \mathbb{R}^{k}\right)$ containing the space of constant mappings $\mathbb{R}^{k}$ and assume we restrict $\mathcal{H}\left(\mathbb{R}^{K}\right)$ to a subgroup $\mathcal{S} H\left(\mathbb{R}^{K}\right)$; then one observes that it is necessary that $\mathcal{S}\left(\mathbb{R}^{K}, \mathbb{R}^{k}\right)$ is a $\left(\mathrm{GL}(k, \mathbb{R}) \times \mathcal{S} H\left(\mathbb{R}^{K}\right)\right)$-submodule such that there is a monomorphism, $\mathcal{S}\left(\mathbb{R}^{K}, \mathbb{R}^{k}\right) \rtimes$ $\left(\mathrm{GL}(k, \mathbb{R}) \times \mathcal{S} H\left(\mathbb{R}^{K}\right)\right) \hookrightarrow \mathcal{S} H\left(\mathbb{R}^{k+K}\right)$. Possible examples of such situations are 
Smooth representations: Define $C^{\infty}\left(\mathbb{R}^{K}, \mathbb{R}^{k}\right)$ to denote the vector space of functions $f: \mathbb{R}^{K} \rightarrow \mathbb{R}^{k}$, which are infinitely many times differentiable and denote by $C^{\infty}\left(\mathbb{R}^{K}\right)$ the group, under composition of maps, of smooth diffeomorphisms of $\mathbb{R}^{K}$. Then by considering $C^{\infty}\left(\mathbb{R}^{K}, \mathbb{R}^{k}\right)$ instead of $\mathcal{M}\left(\mathbb{R}^{K}, \mathbb{R}^{k}\right)$ and replacing $\mathcal{H}\left(\mathbb{R}^{K}\right)$ by $C^{\infty}\left(\mathbb{R}^{K}\right)$, we find the so-called canonical type smooth representations. Here, results analogous to Theorem 2.5 can be formulated (see [CR77] and [LR84]).

Affine representations: Restrict $\mathcal{M}\left(\mathbb{R}^{K}, \mathbb{R}^{k}\right)$ to $\operatorname{Aff}\left(\mathbb{R}^{K}, \mathbb{R}^{k}\right)$ (the vector space of affine mappings) and $\mathcal{H}\left(\mathbb{R}^{K}\right)$ to $\operatorname{Aff}\left(\mathbb{R}^{K}\right)$ (the affine group of $\mathbb{R}^{K}$ ) (see [DI93]).

Polynomial representations: Write $P\left(\mathbb{R}^{K}, \mathbb{R}^{k}\right)$ to refer to the vector space of polynomial mappings $p: \mathbb{R}^{K} \rightarrow \mathbb{R}^{k}$. So $p$ is given by $k$ polynomials in $K$ variables. $P\left(\mathbb{R}^{K}\right)$ will be used to indicate the set of all polynomial diffeomorphisms of $\mathbb{R}^{K}$, with an inverse which is also a polynomial mapping. This is a group where the multiplication is given by composition. It is not hard to verify that $P\left(\mathbb{R}^{K}, \mathbb{R}^{k}\right)$ is a $\operatorname{Aut}\left(\mathbb{Z}^{k}\right) \times P\left(\mathbb{R}^{K}\right)$-module and that the resulting semi-direct product group

$$
P\left(\mathbb{R}^{K}, \mathbb{R}^{k}\right) \rtimes\left(\text { Aut } \mathbb{Z}^{k} \times P\left(\mathbb{R}^{K}\right)\right) \subseteq P\left(\mathbb{R}^{K+k}\right)
$$

by defining $\forall(p, g, h) \in P\left(\mathbb{R}^{K}, \mathbb{R}^{k}\right) \rtimes\left(\right.$ Aut $\left.\mathbb{Z}^{k} \times P\left(\mathbb{R}^{K}\right)\right), \forall(x, y) \in \mathbb{R}^{k+K}$ :

$$
{ }^{(p, g, h)}(x, y)=(g x+p(h(y)), h(y)) .
$$

Restrict $\mathcal{M}\left(\mathbb{R}^{K}, \mathbb{R}^{k}\right)$ to $P\left(\mathbb{R}^{K}, \mathbb{R}^{k}\right)$ and $\mathcal{H}\left(\mathbb{R}^{K}\right)$ to $P\left(\mathbb{R}^{K}\right)$, and we will speak of canonical type polynomial representations.

In each of these restricted situations one now faces existence and uniqueness questions.

Counterexample 2.6. It looks tempting to consider $P_{s}\left(\mathbb{R}^{K}, \mathbb{R}^{k}\right)$, the vector space of polynomial mappings $p: \mathbb{R}^{K} \rightarrow \mathbb{R}^{k}$ which are of total degree $\leq s$. So $p$ is now given by $k$ polynomials in $K$ variables, each of which is of total degree $\leq s$. Use $P_{t}\left(\mathbb{R}^{K}\right)$ to indicate a group consisting only of polynomial diffeomorphisms of $\mathbb{R}^{K}$ of degree $\leq t$. Now remark, however, that $P_{s}\left(\mathbb{R}^{K}, \mathbb{R}^{k}\right)$ will not be a $\operatorname{Aut}\left(\mathbb{Z}^{k}\right) \times P_{t}\left(\mathbb{R}^{K}\right)$ module, as the degrees go up under the group-action.

\section{CANonical type polynomial structures For Nilpotent Groups}

Assume, from now on, that $N$ is a torsion-free, finitely generated nilpotent group of rank $K$ and class $c . N$ is polycyclic and hence has torsion-free filtrations. Of particular interest in our context are filtrations which are central series of length $c$, e.g. the upper central series. Another frequently arising filtration of this kind is obtained as follows. Write $\gamma_{1}(N)=N$ and $\gamma_{i+1}(N)=\left[N, \gamma_{i}(N)\right]$ for the terms of the lower central series of $N$. Now, it is well known that $\sqrt[N]{\gamma_{k}(N)}$ [the so-called root set or isolator of $\gamma_{k}(N)$, i.e. the set of elements having a power in $\gamma_{k}(N)$ ] is a characteristic subgroup of $N$, with $\left[\sqrt[N]{\gamma_{k}(N)}, \sqrt[N]{\gamma_{l}(N)}\right] \subseteq \sqrt[N]{\gamma_{k+l}(N)}$ and $\sqrt[N]{\gamma_{k}(N)} / \sqrt[N]{\gamma_{k+1}(N)}$ torsion-free. We now define $N_{c}=N, N_{c-1}=\sqrt[N]{\gamma_{2}(N)}, \ldots$ $N_{1}=\sqrt[N]{\gamma_{c}(N)}$

So, the torsion-free filtrations we are looking at are central series of the form:

$$
N_{*}: \quad N_{0}=1 \subset N_{1} \subset N_{2} \subset \cdots \subset N_{c-1} \subset N_{c}=N_{c+1}=N .
$$

We will refer to such a filtration, as a torsion-free central series. 
From now on, if we speak of a polynomial representation $\rho: N \rightarrow P\left(\mathbb{R}^{K}\right)$ which is of canonical type, we mean of canonical type with respect to some torsion-free central series.

Referring to Theorem 2.5 and to the fact that we are dealing with a central series, we can state the following corollary.

Corollary 3.1. Assume that $N$ is as above and that $N_{*}$ is some torsion-free central series. If $\rho: N \rightarrow P\left(\mathbb{R}^{K}\right)$ is of canonical type with respect to $N_{*}$, then, $\forall n \in N$ : $\rho(n): \mathbb{R}^{K} \rightarrow \mathbb{R}^{K}: x \mapsto\left(p_{1}^{\gamma}(x), p_{2}^{\gamma}(x), \ldots, p_{c}^{\gamma}(x)\right)$ is such that

$$
p_{i}^{\gamma}(x)=x_{i}+Q_{i}^{n}\left(x_{i+1}, x_{i+2}, \ldots, x_{c}\right),
$$

for some polynomial mapping $Q_{i}^{n}: \mathbb{R}^{K_{i+1}} \rightarrow \mathbb{R}^{k_{i}}$. Moreover, $N_{i}$ acts trivially on $\mathbb{R}^{K_{i+1}}$ and as translations on the ith block $\mathbb{R}^{k_{i}}$, in such a way that these translations generate all of $\mathbb{R}^{k_{i}}$.

\section{Example 3.2.}

$$
\begin{array}{ll}
\text { Let } N:\left\langle a_{1,1}, a_{1,2}, a_{2,1}, a_{2,2}, a_{3,1}, a_{3,2} \|\right. & {\left[a_{3,2}, a_{3,1}\right]=a_{1,1}^{-1} a_{2,1} a_{2,3}^{2}} \\
& {\left[a_{2,1}, a_{3,1}\right]=a_{1,1}^{-2}\left[a_{2,2}, a_{3,2}\right]=a_{1,1}} \\
& {\left[a_{2,3}, a_{2,2}\right]=a_{1,1} \quad\left[a_{2,2}, a_{2,1}\right]=a_{1,1}^{2}} \\
& \text { all other commutators trivial. }
\end{array}
$$

One can check that there is a canonical type representation $\rho: N \longrightarrow P\left(\mathbb{R}^{6}\right)$ of this $N$ given in terms of the images of the generators (the central series used, is suggested by the labeling of the generators):

$$
\begin{aligned}
& \rho\left(a_{1,1}\right)(x)=\left(1+x_{1,1}, x_{2,1}, x_{2,2}, x_{2,3}, x_{3,1}, x_{3,2}\right), \\
& \rho\left(a_{2,1}\right)(x)=\left(x_{1,1}-2 x_{3,1}, 1+x_{2,1}, x_{2,2}, x_{2,3}, x_{3,1}, x_{3,2}\right), \\
& \rho\left(a_{2,2}\right)(x)=\left(x_{1,1}+2 x_{2,1}+x_{3,2}, x_{2,1}, 1+x_{2,2}, x_{2,3}, x_{3,1}, x_{3,2}\right), \\
& \rho\left(a_{2,3}\right)(x)=\left(x_{1,1}+x_{2,2}, x_{2,1}, x_{2,2}, 1+x_{2,3}, x_{3,1}, x_{3,2}\right), \\
& \rho\left(a_{3,1}\right)(x)=\left(x_{1,1}, x_{2,1}, x_{2,2}, x_{2,3}, 1+x_{3,1}, x_{3,2}\right), \\
& \rho\left(a_{3,2}\right)(x)=\left(x_{1,1}+2 x_{2,2} x_{3,1}-x_{3,1}^{2}, x_{2,1}+x_{3,1}, x_{2,2}, x_{2,3}+2 x_{3,1}, x_{3,1}, 1+x_{3,2}\right) .
\end{aligned}
$$

The following theorem, intrinsically due to $\mathrm{Mal}^{\prime} \mathrm{cev}$ ([Mal51]), will imply the existence of canonical type polynomial representations for all torsion-free, f.g. nilpotent groups.

Theorem 3.3. Every connected, simply connected c-step nilpotent Lie group $G$ of dimension $K$ embeds into $P\left(\mathbb{R}^{K}\right)$ in such a way that the image of $G$ consists of polynomials of degree $\leq m$ for $m=\max (1, c-1)$ and so that the action of $G$ on $\mathbb{R}^{K}$ is simply transitive.

Proof. Write $\mathfrak{g}$ for the Lie algebra of $G$. It is well known that there is a one-to-one correspondence between $\mathfrak{g}$ and $G$ via the exponential mapping. We follow $\mathrm{Mal}^{\prime} \mathrm{cev}$ 's ideas to describe the group structure of $G$.

Consider the sequence of Lie subalgebras $\mathfrak{h}^{i}$ of $\mathfrak{g}$ given by the lower central series of $\mathfrak{g}$ in reversed order. So $\mathfrak{h}^{c}=\mathfrak{g}^{(1)}=\mathfrak{g}, \mathfrak{h}^{c-1}=\mathfrak{g}^{(2)}=[\mathfrak{g}, \mathfrak{g}], \ldots$, $\mathfrak{h}^{1}=\mathfrak{g}^{(c)}=\left[\mathfrak{g}, \mathfrak{g}^{(c-1)}\right]$. Choose a basis

$$
A_{1,1}, A_{1,2}, \ldots, A_{1, k_{1}}, A_{2,1}, \ldots, A_{2, k_{2}}, A_{3,1} \ldots, A_{c, k_{c}}
$$

of $\mathfrak{g}$ in such a way that $\mathfrak{h}^{i}$ is the vector space generated by $A_{1,1}, A_{1,2}, \ldots, A_{i, k_{i}}$. Now, introduce a system of coordinates on $G$ by defining for all $g$ of $G$ its coordinate $c(g)$ to be the coordinate of $\log (g)$ with respect to the basis $A_{1,1}, \ldots, A_{c, k_{c}}$. 
Consider $x$ (resp. $y) \in G$, with $\log x=\sum x_{i, j} A_{i, j}\left(\right.$ resp. $\log y=\sum y_{i, j} A_{i, j}$ ). The Campbell-Baker-Hausdorff formula implies that

$$
\begin{aligned}
x y & =\exp \left(\sum x_{i, j} A_{i, j}\right) \exp \left(\sum y_{i, j} A_{i, j}\right) \\
& =\exp \left(\sum\left(x_{i, j}+y_{i, j}\right) A_{i, j}+\frac{1}{2} \sum x_{p, q} y_{r, s}\left[A_{p, q}, A_{r, s}\right]+\cdots\right) .
\end{aligned}
$$

A close examination of the previous expression now shows that the $(i, j)$-th coordinate of the product $x y$ satisfies:

$$
c(x y)_{i, j}=x_{i, j}+y_{i, j}+P_{i, j}\left(x_{i+1,1}, \ldots, x_{c, k_{c}}, y_{i+1,1}, \ldots, y_{c, k_{c}}\right)
$$

where $P_{i, j}$ is a polynomial of total degree $\leq c-i+1$ in all the variables $x_{p, q}$ and $y_{r, s}$ (use the nilpotency of $\mathfrak{g}$ ). Moreover, when regarded as a polynomial in the $x_{p, q}$-variables (resp. $y_{r, s}$-variables) alone, $P_{i, j}$ has degree $\leq c-i$.

So, in terms of the coordinates introduced for $G$, the product is expressed by polynomial functions. We use these functions to define the embedding of $G$ in $P\left(\mathbb{R}^{K}\right)$ as follows:

$$
\rho: G \rightarrow P\left(\mathbb{R}^{K}\right): g \mapsto \rho(g) \text { with }
$$

$\forall y \in \mathbb{R}^{K}:(\rho(g)(y))_{i, j}=c(g)_{i, j}+y_{i, j}+P_{i, j}\left(c(g)_{i+1,1}, \ldots, c(g)_{c, k_{c}}, y_{i+1,1}, \ldots, y_{c, k_{c}}\right)$ It is now easy to see that this $\rho$ indeed defines an action of $G$ on $\mathbb{R}^{K}$ (in fact, it is just left translation in $G$ !), which satisfies the criteria of the statement.

Corollary 3.4. If $G$ admits a lattice $N$, then we may consider the torsion-free central series $N_{*}$ of $N$ determined by the isolators of the lower central series of $N$. If $a_{1,1}, a_{1,2}, \ldots, a_{c, k_{c}}$ denotes a set of generators of $N$ which is compatible with $N_{*}$, we may choose the basis used in the theorem to be determined by

$$
A_{1,1}=\log a_{1,1}, A_{1,2}=\log a_{1,2}, \ldots, A_{c, k_{c}}=\log a_{c, k_{c}} .
$$

Restricting the representation $\rho: G \rightarrow P\left(\mathbb{R}^{K}\right)$ (based on the chosen basis of $\mathfrak{g}$ ) to $N$, we see that we obtain a canonical representation of $N$ into $P\left(\mathbb{R}^{K}\right)$.

In view of this corollary, Counterexample 2.6 is really disappointing. It looks as if the only way out, if one insists on the capability to iterate the set-up, is to go on with the general polynomial (without upper bound degree) situation.

Remark 3.5. The proof of Theorem 3.3 can easily be adjusted to work with more general central series. Therefore, if $G$ admits a lattice $N$, we can find a canonical type polynomial representation of $N$ (of finite degree) with respect to any torsionfree central series $N_{*}$ of $N$.

For technical reasons we need the following (rather well known) lemma, the proof of which is left to the reader.

Lemma 3.6. Let $\Gamma \subseteq \mathbb{R}^{k}$ be any set of elements which span $\mathbb{R}^{k}$ as a vector space, for some $k \in \mathbb{N}_{0}$. Let $p\left(x_{1}, x_{2}, \ldots, x_{k}, x_{k+1}, \ldots, x_{n}\right)$ be a polynomial in $n$ variables for $n \geq k$, with real coefficients. Suppose

$$
p\left(x_{1}+z_{1}, x_{2}+z_{2}, \ldots, x_{k}+z_{k}, x_{k+1}, \ldots, x_{n}\right)=p\left(x_{1}, x_{2}, \ldots, x_{k}, x_{k+1}, \ldots, x_{n}\right)
$$

for all $\left(z_{1}, z_{2}, \ldots, z_{k}\right) \in \Gamma$ and all $\left(x_{1}, x_{2}, \ldots, x_{n}\right) \in \mathbb{R}^{n}$.

Then $p\left(x_{1}, x_{2}, \ldots, x_{k}, x_{k+1}, \ldots, x_{n}\right)$ does not depend on the variables $x_{1}, x_{2}$, $\ldots, x_{k}$. 
Lemma 3.7. Suppose $N$ and $N_{*}$ are as before. For any canonical type polynomial representation of $N$ with respect to $N_{*}$ we have

$$
P\left(\mathbb{R}^{K_{i}}, \mathbb{R}^{k}\right)^{N_{i} / N_{i-1}} \cong P\left(\mathbb{R}^{K_{i+1}}, \mathbb{R}^{k}\right)
$$

for all $i, 1 \leq i<c$, as $N / N_{i}-$ module.

Proof. Since $P\left(\mathbb{R}^{K_{i}}, \mathbb{R}^{k}\right)$ is an $N / N_{i-1}$-module via ${ }^{h} p=p \circ \rho_{i-1}(h)^{-1}$, it makes sense to speak about $P\left(\mathbb{R}^{K_{i}}, \mathbb{R}^{k}\right)^{N_{i} / N_{i-1}}$. Let $p(x) \in P\left(\mathbb{R}^{K_{i}}, \mathbb{R}^{k}\right)$. Then it is easy to see that ${ }^{z} p(x)=p(x)$ for all $z \in N_{i} / N_{i-1}$ and $x \in \mathbb{R}$ if and only if $p(x)$ is independent of the variables $x_{i, 1}, x_{i, 2}, \ldots x_{i, k_{i}}$ (see Lemma 3.6). Therefore, we may identify $p(x)$ with an element of $P\left(\mathbb{R}^{K_{i+1}}, \mathbb{R}^{k}\right)$. This identification $P\left(\mathbb{R}^{K_{i+1}}, \mathbb{R}^{k}\right) \hookrightarrow$ $P\left(\mathbb{R}^{K_{i}}, \mathbb{R}^{k}\right)$ is of course compatible with the $N / N_{i}$-action.

Theorem 3.8. Let $E$ be a group containing a finitely generated torsion-free nilpotent group $N$ of rank $K$ as a subgroup of finite index. Let $\varphi: E \longrightarrow \operatorname{Aut}\left(\mathbb{Z}^{k}\right)$ be any morphism with $\varphi(N)=1$ and let $\rho: E \longrightarrow P\left(\mathbb{R}^{K}\right)$ be any representation restricting to a canonical type polynomial representation of $N$, with respect to some torsion-free central series. With a E-module structure on $P\left(\mathbb{R}^{K}, \mathbb{R}^{k}\right)$ via

$$
{ }^{h} p=\varphi(h) \circ p \circ \rho(h)^{-1}, \quad p \in P\left(\mathbb{R}^{K}, \mathbb{R}^{k}\right), h \in E,
$$

we have $H^{i}\left(E, P\left(\mathbb{R}^{K}, \mathbb{R}^{k}\right)\right)=0$ for all $i \geq 1$.

Proof. Because $P\left(\mathbb{R}^{K}, \mathbb{R}^{k}\right)$ is a vector space and $N$ is of finite index in $E$, we know that the restriction map

$$
H^{i}\left(E, P\left(\mathbb{R}^{K}, \mathbb{R}^{k}\right)\right) \stackrel{\text { res }}{\rightarrow} H^{i}\left(N, P\left(\mathbb{R}^{K}, \mathbb{R}^{k}\right)\right)
$$

is injective. Therefore, it is enough to prove the statement for the case when $E=N$. First we prove that $H^{1}\left(N_{1}, P\left(\mathbb{R}^{K}, \mathbb{R}^{k}\right)\right)=0$. Observe that $N_{1}$ is a free abelian group, $\varphi: N_{1} \rightarrow \operatorname{Aut}\left(\mathbb{Z}^{k}\right)$ is trivial and $\rho: N_{1} \rightarrow P\left(\mathbb{R}^{K}\right)$ maps into pure translations, since $\rho: N \rightarrow P\left(\mathbb{R}^{K}\right)$ is of canonical type. Let $P_{s}\left(\mathbb{R}^{K}, \mathbb{R}^{k}\right)$ be the group of all polynomial maps of degree $\leq s$. Since $\rho: N_{1} \rightarrow P\left(\mathbb{R}^{K}\right)$ has image in $\operatorname{Aff}\left(\mathbb{R}^{K}\right), P_{s}\left(\mathbb{R}^{K}, \mathbb{R}^{k}\right)$ is an $N_{1}$-module. (Note that, for general $\rho, \operatorname{Aff}\left(\mathbb{R}^{K}\right)$ cannot be made an $N_{1}$-module.) Now we can apply Theorem 2.5 of [IL90] which states that the homomorphism $H^{1}\left(N_{1}, P_{s}\left(\mathbb{R}^{K}, \mathbb{R}^{k}\right)\right) \rightarrow H^{1}\left(N_{1}, P_{s+1}\left(\mathbb{R}^{K}, \mathbb{R}^{k}\right)\right)$, induced from the inclusion homomorphism $P_{s}\left(\mathbb{R}^{K}, \mathbb{R}^{k}\right) \rightarrow P_{s+1}\left(\mathbb{R}^{K}, \mathbb{R}^{k}\right)$ of the coefficient, is trivial. Consequently, $H^{1}\left(N_{1}, P\left(\mathbb{R}^{K}, \mathbb{R}^{k}\right)\right)=0$. To show $H^{i}\left(N_{1}, P\left(\mathbb{R}^{K}, \mathbb{R}^{k}\right)\right)=0$ for $i>1$, we use induction on the rank of $N_{1}$. For $N_{1}=\mathbb{Z}^{1}, H^{i}\left(N_{1}, P\left(\mathbb{R}^{K}, \mathbb{R}^{k}\right)\right)=0$ for $i>1$, as $\mathbb{Z}^{1}$ has cohomological dimension one. Suppose $H^{i}\left(\mathbb{Z}^{l-1}, P\left(\mathbb{R}^{K}, \mathbb{R}^{k}\right)\right)=$ 0 for all $i \geq 1$. Without loss of generality we assume that $\mathbb{Z}^{l}$ has a normal subgroup $\mathbb{Z}$, with torsion-free quotient $\mathbb{Z}^{l} / \mathbb{Z}$ and acting only on the first component of $\mathbb{R}^{K}$. As in Lemma 3.7 we find that $P\left(\mathbb{R}^{K}, \mathbb{R}^{k}\right)^{\mathbb{Z}} \cong P\left(\mathbb{R}^{K-1}, \mathbb{R}^{k}\right)$ as $\mathbb{Z}^{l} / \mathbb{Z}=\mathbb{Z}^{l-1}$ modules. Since $H^{i}\left(\mathbb{Z}, P\left(\mathbb{R}^{K}, \mathbb{R}^{k}\right)\right)=0$ for all $i$, the restriction-inflation exact sequence yields isomorphisms:

$$
0=H^{i}\left(\mathbb{Z}^{l-1}, P\left(\mathbb{R}^{K-1}, \mathbb{R}^{k}\right)\right) \cong H^{i}\left(\mathbb{Z}^{l}, P\left(\mathbb{R}^{K}, \mathbb{R}^{k}\right)\right) .
$$

This proves (something more than) the theorem for torsion-free abelian groups. This generalises Theorem 2.5 of [IL90].

Now we consider any finitely generated nilpotent group $N$ of nilpotency class $c>1$ and we assume the theorem holds for groups of lower nilpotency class. By the above we know that $H^{i}\left(N_{1}, P\left(\mathbb{R}^{K}, \mathbb{R}^{k}\right)\right)=0$ for $i \geq 1$. For the $N$-module 
$P\left(\mathbb{R}^{K}, \mathbb{R}^{k}\right)$ with the normal subgroup subgroup $N_{1}$ of $N$, we apply Lyndon-Serre spectral sequence (the restriction-inflation exact sequence) to get isomorphisms:

$$
H^{i}\left(N, P\left(\mathbb{R}^{K}, \mathbb{R}^{k}\right)\right) \cong H^{i}\left(N / N_{1}, P\left(\mathbb{R}^{K}, \mathbb{R}^{k}\right)^{N / N_{1}}\right) \cong H^{i}\left(N / N_{1}, P\left(\mathbb{R}^{K_{2}}, \mathbb{R}^{k}\right)\right),
$$

where the second isomorphism is due to Lemma 3.7. Finally the last term is trivial by induction hypothesis. This completes the proof.

Corollary 3.9. Let $E$ be as above and assume $\varphi: E \rightarrow \operatorname{Aut}\left(P\left(\mathbb{R}^{K}, \mathbb{R}^{k}\right)\right)$ is any representation, which is conjugated inside $\operatorname{Aut}\left(P\left(\mathbb{R}^{K}, \mathbb{R}^{k}\right)\right)$, to a canonical type polynomial representation $\rho: E \rightarrow P\left(\mathbb{R}^{K}\right) \rightarrow \operatorname{Aut}\left(P\left(\mathbb{R}^{K}, \mathbb{R}^{k}\right)\right)$, then

$$
H_{\varphi}^{i}\left(E, P\left(\mathbb{R}^{K}, \mathbb{R}^{k}\right)\right)=0, \quad \forall i>0 .
$$

Lemma 3.10. Let $E$ be a group containing a torsion-free, finitely generated nilpotent group $N$ as a normal subgroup of finite index. If $\rho: E \rightarrow P\left(\mathbb{R}^{K}\right)$ is a representation of $E$, restricting to a canonical type polynomial representation of $N$, with respect to a torsion-free central series $N_{*}$, then $\rho$ itself is canonical, with respect to $E_{*}$, where $E_{*}$ is given by:

$E_{*}: \quad E_{0}=N_{0}=1 \subset E_{1}=N_{1} \subset \cdots \subset E_{c-1}=N_{c-1} \subset E_{c}=N_{c} \subset E_{c+1}=E$.

Proof. It is enough to show that, for $e \in E, \rho(e)$ is such that

$$
\begin{aligned}
\rho(e) & : \mathbb{R}^{K} \longrightarrow \mathbb{R}^{K} \\
& : x=\left(x_{1,1}, x_{1,2}, \ldots, x_{1, k_{1}}, x_{2,1}, \ldots, x_{i, j}, \ldots, x_{c, k_{c}}\right) \mapsto \rho(e)(x),
\end{aligned}
$$

with $\rho(e)(x)=\left(P_{1,1}^{e}(x), \ldots, P_{i, j}^{e}(x), \ldots, P_{c, k_{c}}^{e}(x)\right)$, and $P_{i, j}^{e}(x)$ is a polynomial depending only on the variables $x_{p, q}$ with $p \geq i$.

First we show that $P_{i, j}^{e}(x)$ does not depend on $x_{1, q}$, if $i>1$. Let $z$ be any element of $N_{1}$, then there exists a $z^{\prime} \in N_{1}$ such that

$$
e z=z^{\prime} e \text {. }
$$

We will look at the effect of both sides of (3) on a general element $x$ with coordinates $\left(x_{1,1}, x_{1,2}, \ldots, x_{c, k_{c}}\right)$.

Remember that $z$ (resp. $z^{\prime}$ ) acts on $\mathbb{R}^{K}$ by some translation on the first $k_{1}$ components, say by $\left(z_{1,1}, \ldots, z_{1, k_{1}}\right)$ (resp. $\left.\left(z_{1,1}^{\prime}, \ldots, z_{1, k_{1}}^{\prime}\right)\right)$. On the one hand we have that

$$
\begin{aligned}
{ }^{e z} x & ={ }^{e}\left({ }^{z} x\right) \\
& ={ }^{e}\left(z_{1,1}+x_{1,1}, \ldots, z_{1, k_{1}}+x_{1, k_{1}}, x_{2,1}, x_{2,2}, \ldots, x_{c, k_{c}}\right) \\
& =\left(P_{1,1}^{e}\left(z_{1,1}+x_{1,1}, \ldots, x_{c, k_{c}}\right), \ldots, P_{c, k_{c}}^{e}\left(z_{1,1}+x_{1,1}, \ldots, x_{c, k_{c}}\right)\right)
\end{aligned}
$$

while, on the other hand, we see that

$$
\begin{aligned}
{ }^{e z} x={ }^{z^{\prime} e} x & ={ }^{\prime}\left({ }^{e} x\right) \\
& =\left(z_{1,1}^{\prime}, \ldots, z_{1, k_{1}}^{\prime}, 0 \ldots, 0\right)+\left(P_{1,1}^{e}(x), \ldots, P_{c, k_{c}}^{e}(x)\right) \\
& =\left(P_{1,1}^{e}(x)+z_{1,1}^{\prime}, \ldots, P_{1, k_{1}}^{e}+z_{1, k_{1}}^{\prime}, P_{2,1}^{e}(x), \ldots, P_{c, k_{c}}^{e}(x)\right) .
\end{aligned}
$$

These two computations result in $\forall i>1, \forall z \in N_{1}$ :

$$
\begin{gathered}
P_{i, j}^{e}\left(z_{1,1}+x_{1,1}, \ldots, z_{1, k_{1}}+x_{1, k_{1}}, x_{2,1}, \ldots, x_{c, k_{c}}\right) \\
=P_{i, j}^{e}\left(x_{1,1}, \ldots, x_{1, k_{1}}, x_{2,1}, \ldots, x_{c, k_{c}}\right) .
\end{gathered}
$$

Since the set of all $\left(z_{1,1}, \ldots, z_{1, k_{1}}\right)$ forms a lattice of $\mathbb{R}^{k_{1}}$, we conclude that the polynomial $P_{i, j}^{e}\left(x_{1,1}, \ldots, x_{c, k_{c}}\right)$ has to be independent of the coordinates $x_{1, q}$ $\left(1 \leq q \leq k_{1}\right)$. 
The same technique can now be used to proceed this proof. One shows by induction on $p$ that $P_{i, j}^{e}$ is independent of $x_{p, q}$ if $i>p$.

Remark 3.11. We will call a filtration $E_{*}$ as in the lemma above a torsion-free pseudo-central series of $E$. If $E$ is virtually nilpotent as above, then, if we consider a canonical type representation we will always mean "canonical with respect to a torsion-free pseudo-central series of $E$ ".

We finish this section with the following lemma:

Lemma 3.12. Let $E$ be a virtually abelian group containing a group $\mathbb{Z}^{k}$ as a normal subgroup of finite index. Then a canonical type polynomial representation is an affine representation.

Proof. Let $\rho: E \rightarrow P\left(\mathbb{R}^{k}\right)$ be the canonical type polynomial representation. We know that the abelian normal subgroup acts on $\mathbb{R}^{k}$ via translations:

$$
{ }^{z} x=z+x \quad \forall z \in \mathbb{Z}^{k} \text { and } \forall x \in \mathbb{R}^{k} .
$$

There is a homomorphism $\varphi: E \rightarrow \operatorname{Aut}\left(\mathbb{Z}^{k}\right)$ such that $e z e^{-1}=\varphi(e) z$. Fix an element $e$ and assume $\varphi(e)$ is given by a matrix $\left(\alpha_{i, j}\right)_{1 \leq i, j \leq k}$. We denote $\rho(e)=$ $\left(P_{1}(x), P_{2}(x), \ldots, P_{k}(x)\right)$, where the $P_{i}(x)$ are some polynomials in $k$ variables. For any element $x=\left(x_{1}, x_{2}, \ldots, x_{k}\right)$ and any $z=\left(z_{1}, z_{2}, \ldots, z_{k}\right) \in \mathbb{Z}^{k}$ we have that

$$
\begin{aligned}
& { }^{e z} x=(\varphi(e) z) e x \\
& { }^{e}\left(x_{1}+z_{1}, \ldots, x_{k}+z_{k}\right)=\varphi(e) z+\left(P_{1}(x), \ldots, P_{k}(x)\right) \\
& \Downarrow \\
& P_{i}\left(x_{1}+z_{1}, \ldots, x_{k}+z_{k}\right)=\alpha_{i, 1} z_{1}+\cdots+\alpha_{i, k} z_{k}+P_{i}\left(x_{1}, \ldots, x_{k}\right) \text {. } \\
& (1 \leq i \leq k)
\end{aligned}
$$

Now, consider the polynomial

$$
Q(x)=P_{i}(x)-\alpha_{i, 1} x_{1}-\cdots-\alpha_{i, k} x_{k}-P_{i}(0) .
$$

The previous computation shows that $Q(z)=0 \forall z \in \mathbb{Z}^{k}$. This implies that $Q(x)=$ 0 or that $P_{i}(x)$ is a polynomial of degree 1 .

\section{EXISTENCE AND UNiQUENESS OF POLYNOMIAL MANIFOLDS}

In this section we make intensive use of the basic facts of the theory of Seifert Fiber Space constructions. We refer to [Lee83] and [CR77] for the full details concerning this concept.

With a polynomial manifold of dimension $n$ we mean a quotient manifold of the form $E \backslash \mathbb{R}^{n}$ where $E$ is a group acting freely and properly discontinuously on $\mathbb{R}^{n}$ via a polynomial action $\rho: E \rightarrow P\left(\mathbb{R}^{n}\right)$. If the image of $\rho$ consists only of polynomials of degree $\leq s$, we say that the manifold $E \backslash \mathbb{R}^{n}$ is of degree $\leq s$. Whenever we use the term manifold, we will mean a closed manifold.

Theorem 4.1. (Existence) Let $N$ be a torsion-free, finitely generated nilpotent group of rank $K$. For any torsion-free central series $N_{*}$, there exists a canonical type polynomial representation $\rho: N \rightarrow P\left(\mathbb{R}^{K}\right)$.

(Uniqueness) If $\rho_{1}, \rho_{2}$ are two such polynomial representations of $N$ into $P\left(\mathbb{R}^{K}\right)$ with respect to the same $N_{*}$, there exists a polynomial map $p \in P\left(\mathbb{R}^{K}\right)$ such that $\rho_{2}=p^{-1} \circ \rho_{1} \circ p$. Consequently, the manifolds $\rho_{1}(N) \backslash \mathbb{R}^{K}$ and $\rho_{2}(N) \backslash \mathbb{R}^{K}$ are "polynomially diffeomorphic". 
Proof. (Existence) The existence is already covered by Corollary 3.4. Nevertheless, it is useful to present an alternative proof here; in fact, this proof, as it is based on a cohomological argument, has the advantage of generalising to the virtual nilpotent situation and offering a set-up allowing us to treat the uniqueness. We proceed by induction on the nilpotency class $c$ of $N$. If $N$ is abelian, then the existence is well known. Now, suppose that $N$ is of class $c>1$ and the existence is guaranteed for lower nilpotency classes. Using the induction hypothesis, the group $N / N_{1}$ can be furnished with a canonical type polynomial representation

$$
\bar{\rho}: N / N_{1} \rightarrow P\left(\mathbb{R}^{K_{2}}\right) .
$$

We obtain an embedding $i: N_{1} \cong \mathbb{Z}^{k_{1}} \rightarrow P\left(\mathbb{R}^{K_{2}}, \mathbb{R}^{k_{1}}\right)$ if we define $i(z): \mathbb{R}^{K_{2}} \rightarrow$ $\mathbb{R}^{k_{1}}: x \mapsto z$. We are looking for a map $\rho$ making the following diagram commutative:

$$
\begin{aligned}
& 1 \rightarrow \quad N_{1} \quad \rightarrow \quad N \quad N \quad \rightarrow \quad N / N_{1} \quad \rightarrow 1 \\
& \downarrow i \quad \downarrow \rho \quad \downarrow \varphi \times \bar{\rho} \\
& 1 \rightarrow P\left(\mathbb{R}^{K_{2}}, \mathbb{R}^{k_{1}}\right) \rightarrow P \rtimes(A \times P) \rightarrow \text { Aut } \mathbb{Z}^{k_{1}} \times P\left(\mathbb{R}^{K_{2}}\right) \rightarrow 1 \\
& P\left(\mathbb{R}^{K}\right)
\end{aligned}
$$

where $P \rtimes(A \times P)$ denotes $P\left(\mathbb{R}^{K_{2}}, \mathbb{R}^{k_{1}}\right) \rtimes\left(\right.$ Aut $\left.\mathbb{Z}^{k_{1}} \times P\left(\mathbb{R}^{K_{2}}\right)\right)$ and $\varphi: N / N_{1} \rightarrow$ Aut $\mathbb{Z}^{k_{1}}=$ Aut $N_{1}$ denotes the morphism induced by the extension $1 \rightarrow N_{1} \rightarrow$ $N \rightarrow N / N_{1} \rightarrow 1$.

The existence of such a map $\rho$ is now guaranteed by the surjectiveness of $\delta$ in the long exact cohomology sequence

$$
\begin{gathered}
\cdots \rightarrow \underset{\|}{\|} \rightarrow H^{1}\left(N / N_{1}, P\left(\mathbb{R}^{K_{2}}, \mathbb{R}^{k_{1}}\right) / \mathbb{Z}^{k_{1}}\right) \stackrel{\delta}{\rightarrow} H^{2}\left(N / N_{1}, \mathbb{Z}^{k_{1}}\right) \rightarrow \underset{\|}{ } \rightarrow \cdots \\
H^{1}\left(N / N_{1}, P\left(\mathbb{R}^{K_{2}}, \mathbb{R}^{k_{1}}\right)\right) \quad\left(\text { Theorem 3.8) } \quad H^{2}\left(N / N_{1}, P\left(\mathbb{R}^{K_{2}}, \mathbb{R}^{k_{1}}\right)\right)\right.
\end{gathered}
$$

(Uniqueness) Again we proceed by induction on the nilpotency class $c$ of $N$. For $c=1$ the result is again well known. Indeed, two canonical type representations of a virtually abelian group are even known to be affinely conjugated.

So we suppose that $N$ is of class $c>1$ and that the theorem holds for smaller nilpotency classes. The representations $\rho_{1}, \rho_{2}$ induce two canonical type polynomial representations

$$
\overline{\rho_{1}}, \overline{\rho_{2}}: N / N_{1} \rightarrow \mathbb{R}^{K_{2}}
$$

By the induction hypothesis, there exists a polynomial map $\bar{q}: \mathbb{R}^{K_{2}} \rightarrow \mathbb{R}^{K_{2}}$ such that

$$
\bar{\rho}_{2}=\bar{q}^{-1} \circ \bar{\rho}_{1} \circ \bar{q} .
$$

Lift this $\bar{q}$ to a polynomial map $q$ of $\mathbb{R}^{K_{1}}$ as follows:

$$
\forall x \in \mathbb{R}^{k_{1}}, \forall y \in \mathbb{R}^{K_{2}}: q: \mathbb{R}^{K_{1}} \rightarrow \mathbb{R}^{K_{1}}:(x, y) \mapsto q(x, y)=(x, \bar{q}(y)) .
$$

When restricted to $N_{1}, \rho_{1}$ and $\rho_{2}$ are mapping elements onto pure translations of $\mathbb{R}^{k_{1}}$. We know that there exists an affine mapping $\tilde{A}: \mathbb{R}^{k_{1}} \rightarrow \mathbb{R}^{k_{1}}$ for which

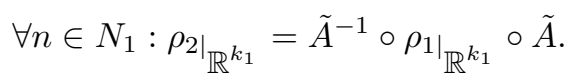


Extend $\tilde{A}$ to an affine mapping of $\mathbb{R}^{K}$ by defining

$$
\forall x \in \mathbb{R}^{k_{1}}, \forall y \in \mathbb{R}^{K_{2}}: A: \mathbb{R}^{K_{1}} \rightarrow \mathbb{R}^{K_{1}}:(x, y) \mapsto A(x, y)=(\tilde{A}(x), y) .
$$

Let us denote $\psi=A^{-1} \circ q^{-1} \circ \rho_{1} \circ q \circ A$. Then we see that $\psi$ and $\rho_{2}$ are two canonical type polynomial representations of $N$, which coincide with each other on $N_{1}$ and which induce the same representation of $N / N_{1}$. This means that $\psi$ and $\rho_{2}$ can be seen as the result of a Seifert construction with respect to the same data (cf. the commutative diagram above).

Now we use the injectiveness of $\delta$ in (4), which implies that $\psi$ and $\rho_{2}$ are conjugated to each other by an element $r \in P\left(\mathbb{R}^{K_{2}}, \mathbb{R}^{k_{1}}\right)$ (seen as an element of $P\left(\mathbb{R}^{K}\right)$ !). So we may conclude that

$$
\rho_{2}=r^{-1} \circ \psi \circ r=r^{-1} \circ A^{-1} \circ q^{-1} \circ \rho_{1} \circ q \circ A \circ r=p^{-1} \circ \rho_{1} \circ p
$$

if we take $p=q \circ A \circ r$.

Corollary 4.2. Any canonical polynomial representation with respect to some torsion-free central series of $N$ is polynomially conjugated to a canonical type polynomial representation of $N$ of degree $\leq s$, where $s=\max (1, c-1)$. Consequently, for each $N$ there is a well defined number

$m(N)=$

$\operatorname{Min}\{s \in \mathbb{N}|| N$ is fundamental group of a polynomial manifold of degree $\leq s\}$.

Corollary 4.3. Since any canonical type polynomial representation $\rho$ of $N$ is polynomially conjugated to a polynomial representation of finite degree, $\rho$ itself has to be of finite degree.

By replacing $N$ by a group $E$ which contains $N$ as a normal subgroup of finite index, and noting that

$$
H^{i}\left(E, P\left(\mathbb{R}^{K}, \mathbb{R}^{k}\right)\right) \stackrel{\text { res }}{\longrightarrow} H^{i}\left(N, P\left(\mathbb{R}^{K}, \mathbb{R}^{k}\right)\right)
$$

is injective, we get the following

Corollary 4.4. Let $E$ be a group containing a normal subgroup $N$, which is torsionfree, finitely generated of rank $K$, nilpotent of class $c$ and of finite index in $E$ and fix a torsion-free pseudo-central series $E_{*}$ of $E$. Then there exists a canonical type polynomial representation of $E$ with respect to this series.

Moreover, if $\rho_{1}, \rho_{2}$ are two canonical type polynomial representations of $E$ into $P\left(\mathbb{R}^{K}\right)$, with respect to $E_{*}$, then there exists a polynomial map $p \in P\left(\mathbb{R}^{K}\right)$ such that $\rho_{2}=p^{-1} \circ \rho_{1} \circ p$. In case $E$ is torsion-free this means that the manifolds $\rho_{1}(E) \backslash \mathbb{R}^{K}$ and $\rho_{2}(E) \backslash \mathbb{R}^{K}$ are "polynomially diffeomorphic".

Corollary 4.5. Suppose $E$ acts properly discontinuously on $\mathbb{R}^{K}$, via $\varphi: E \rightarrow$ $\operatorname{Aff}\left(\mathbb{R}^{K}\right)$ and suppose also that there exists a canonical type affine representation $\rho: E \rightarrow \operatorname{Aff}\left(\mathbb{R}^{K}\right)$, then

$$
H_{\varphi}^{i}\left(E, P\left(\mathbb{R}^{K}, \mathbb{R}^{k}\right)\right)=0, \quad \forall i>0 .
$$

Proof. The results of [FG83] imply that $\varphi$ and $\rho$ are conjugated inside $P\left(\mathbb{R}^{K}\right)$. Hence Corollary 3.9 can be applied.

Corollary 4.6. Suppose $E$ is a virtually 3 -step nilpotent group of rank $K$, acting properly discontinuously on $\mathbb{R}^{K}$, via $\varphi: E \rightarrow \operatorname{Aff}\left(\mathbb{R}^{K}\right)$, then

$$
H_{\varphi}^{i}\left(E, P\left(\mathbb{R}^{K}, \mathbb{R}^{k}\right)\right)=0, \quad \forall i>0 .
$$


Proof. We can apply the previous corollary, since any virtually 3-step nilpotent group admits a canonical type affine representation ([Lee83]).

Remark 4.7. Recently Hartl ([Har91]) proved that for $N$ finitely generated, torsionfreenilpotent of class $c, H^{2}\left(N, \mathbb{Z}^{k}\right)$ is polynomial of degree $\leq c$. It, once again, looks tempting to investigate its relation with working in $P_{c}\left(\mathbb{R}^{K}, \mathbb{R}^{k}\right)$. However, Counterexample 2.6 once again obstructs an elaboration of this approach.

In view of Milnor's problem mentioned in the beginning and recent counterexamples obtained by Benoist ([Ben92]) and Burde and Grunewald ([BG93]), it looks interesting to introduce the following concept.

Definition 4.8. For $N$ finitely generated, torsion-free nilpotent of class $c$ and rank $K$, we define the affine defect of $N, d(N)$, to be

$$
d(N)=m(N)-1
$$

Lemma 4.9. For $N$ as above, we can say that

1. $d(N)=0$ iff $N$ is fundamental group of a complete affinely flat compact manifold.

2. $0 \leq d(N)=m(N)-1 \leq \operatorname{Max}\{0, c-2\}$. This follows at once from the results above.

3. If $c \leq 3$ or $K \leq 7$, then $d(N)=0$. The first is proved in [Sch74]; for the second, we refer to [Ben92].

The affine defect $d(N)$ measures the obstruction for $N$ to be fundamental group of a complete affinely flat compact manifold. As we will show in our last section, the counter-examples to Milnor's question studied so far are of weakest possible affine defect.

Remark 4.10. Corollary 4.4 is a generalization of Theorem 1.20 in [FG83] and of Theorem 4.1 in [IL90]. In this second paper, the authors are concerned with canonical type affine representations (called "special" there), with respect to the upper central series. They claim that we can take the polynomial to conjugate with, of degree $\leq c$ !. Certainly there is a polynomial conjugation, but the degree does not seem to be right. We indicate a gap in the proof of Theorem 4.1 of [IL90].

First notice that, in the case where $c=1$, the polynomial needed to conjugate two canonical type affine representations should be taken of degree 1 . The real gap is in the induction argument in [IL90]. Indeed, an induction argument assumes that, after conjugation by a polynomial on the $N / N_{1}=N / Z(N)$-level, the result on the $N$-level is again a canonical type affine representation. Of course, the conjugation does not touch the canonical type character of the representation. However, as the subsequent example shows, one might obtain a polynomial representation which stays outside the affine group.

Example 4.11. Let $N$ be the following finitely generated torsion-free 3 -step nilpotent group:

$$
N:\left\langle a_{1}, a_{2}, b, c \|\left[a_{2}, a_{1}\right]=b,\left[b, a_{1}\right]=c,\left[b, a_{2}\right]=\left[c, a_{1}\right]=\left[c, a_{2}\right]=[c, b]=1\right\rangle .
$$


We propose the following two canonical type affine representations of $N$ (upper central series), where we indicate only the images of the generators:

$$
\begin{gathered}
\rho_{1}\left(a_{1}\right)=\left(\left(\begin{array}{cccc}
1 & -1 & 0 & 1 \\
0 & 1 & 0 & -1 \\
0 & 0 & 1 & 0 \\
0 & 0 & 0 & 1
\end{array}\right),\left(\begin{array}{l}
0 \\
0 \\
1 \\
0
\end{array}\right)\right) \rho_{1}\left(a_{2}\right)=\left(\left(\begin{array}{llll}
1 & 0 & 0 & 0 \\
0 & 1 & 0 & 0 \\
0 & 0 & 1 & 0 \\
0 & 0 & 0 & 1
\end{array}\right),\left(\begin{array}{l}
0 \\
0 \\
0 \\
1
\end{array}\right)\right) \\
\rho_{1}(b)=\left(\left(\begin{array}{llll}
1 & 0 & 0 & 0 \\
0 & 1 & 0 & 0 \\
0 & 0 & 1 & 0 \\
0 & 0 & 0 & 1
\end{array}\right),\left(\begin{array}{l}
0 \\
1 \\
0 \\
0
\end{array}\right)\right) \quad \rho_{1}(c)=\left(\left(\begin{array}{llll}
1 & 0 & 0 & 0 \\
0 & 1 & 0 & 0 \\
0 & 0 & 1 & 0 \\
0 & 0 & 0 & 1
\end{array}\right),\left(\begin{array}{l}
1 \\
0 \\
0 \\
0
\end{array}\right)\right)
\end{gathered}
$$

and

$$
\begin{gathered}
\rho_{2}\left(a_{1}\right)=\left(\left(\begin{array}{cccc}
1 & -\frac{2}{3} & 0 & 0 \\
0 & 1 & 0 & -\frac{1}{2} \\
0 & 0 & 1 & 0 \\
0 & 0 & 0 & 1
\end{array}\right),\left(\begin{array}{l}
0 \\
0 \\
1 \\
0
\end{array}\right)\right) \rho_{2}\left(a_{2}\right)=\left(\left(\begin{array}{llll}
1 & 0 & 0 & 0 \\
0 & 1 & \frac{1}{2} & 0 \\
0 & 0 & 1 & 0 \\
0 & 0 & 0 & 1
\end{array}\right),\left(\begin{array}{l}
0 \\
0 \\
0 \\
1
\end{array}\right)\right) \\
\rho_{2}(b)=\left(\left(\begin{array}{cccc}
1 & 0 & \frac{1}{3} & 0 \\
0 & 1 & 0 & 0 \\
0 & 0 & 1 & 0 \\
0 & 0 & 0 & 1
\end{array}\right),\left(\begin{array}{l}
\frac{2}{3} \\
1 \\
0 \\
0
\end{array}\right)\right) \quad \rho_{2}(c)=\left(\left(\begin{array}{llll}
1 & 0 & 0 & 0 \\
0 & 1 & 0 & 0 \\
0 & 0 & 1 & 0 \\
0 & 0 & 0 & 1
\end{array}\right),\left(\begin{array}{l}
1 \\
0 \\
0 \\
0
\end{array}\right)\right) .
\end{gathered}
$$

The presentation for $N / Z(N)$ can be read from the presentation of $N$, by simply replacing all $c$ 's by 1 . The induced canonical type affine representations $\bar{\rho}_{1}, \bar{\rho}_{2}$ : $N / Z(N) \rightarrow \operatorname{Aff}\left(\mathbb{R}^{3}\right)$ are also easy to write down:

- In the linear part of $\rho_{i}(i=1,2)$, we discard the first column and the first row.

- In the translational part, we omit the first term.

Following the general theory of Seifert Fiber Spaces and the proof of Theorem 4.1 in [IL90] or of Theorem 4.4 we find that there must exist a polynomial mapping $p(x)$ in $P\left(\mathbb{R}^{2}, \mathbb{R}\right)$ such that

$$
\bar{\rho}_{2}=p^{-1} \circ \bar{\rho}_{1} \circ p
$$

when $p(x)$ is seen as an element of $P\left(\mathbb{R}^{2}, \mathbb{R}\right) \rtimes P\left(\mathbb{R}^{2}\right)$ or as an element of $P\left(\mathbb{R}^{3}\right)$. Some elementary computations show that the only possible $p$ 's are of the form:

$$
p: \mathbb{R}^{3} \rightarrow \mathbb{R}^{3}:\left(\begin{array}{c}
z \\
y \\
x
\end{array}\right) \mapsto\left(\begin{array}{c}
z-\frac{x y}{2}+r \\
y \\
x
\end{array}\right)
$$

where $r$ can be any real number. If we now lift this $p$ to a polynomial mapping of $\mathbb{R}^{4}$, say

$$
q: \mathbb{R}^{4} \rightarrow \mathbb{R}^{4}:\left(\begin{array}{c}
t \\
z \\
y \\
x
\end{array}\right) \mapsto\left(\begin{array}{c}
t \\
z-\frac{x y}{2}+r \\
y \\
x
\end{array}\right)
$$

we find that

$$
q^{-1} \rho_{1}\left(a_{1}\right) q: \mathbb{R}^{4} \rightarrow \mathbb{R}^{4}:\left(\begin{array}{c}
t \\
z \\
y \\
x
\end{array}\right) \mapsto\left(\begin{array}{c}
t+x-z+\frac{x y}{2}-r \\
z-\frac{x}{2} \\
y+1 \\
x
\end{array}\right)
$$


which is clearly not affine. Moreover, the same can be said about any lift of $p$ of the form

$$
q: \mathbb{R}^{4} \rightarrow \mathbb{R}^{4}:\left(\begin{array}{c}
t \\
z \\
y \\
x
\end{array}\right) \mapsto\left(\begin{array}{c}
m(x, y, z, t) \\
z-\frac{x y}{2}+r \\
y \\
x
\end{array}\right)
$$

where $m(x, y, z, t)$ denotes some polynomial of degree $\leq 2$ in the four variables.

Finally, we indicate how $\rho_{1}$ and $\rho_{2}$ can be seen to be conjugated by a polynomial. Consider

$$
p: \mathbb{R}^{4} \rightarrow \mathbb{R}^{4}:\left(\begin{array}{c}
t \\
z \\
y \\
x
\end{array}\right) \mapsto\left(\begin{array}{c}
t+\frac{x y}{3}+\frac{x y^{2}}{6}-\frac{2 z}{3}-\frac{y z}{3} \\
z-\frac{x y}{2} \\
y \\
x
\end{array}\right)
$$

Then one can see that

$$
p^{-1}: \mathbb{R}^{4} \rightarrow \mathbb{R}^{4}:\left(\begin{array}{c}
t \\
z \\
y \\
x
\end{array}\right) \mapsto\left(\begin{array}{c}
t+\frac{2 z}{3}+\frac{y z}{3} \\
z+\frac{x y}{2} \\
y \\
x
\end{array}\right)
$$

and some calculations then result in

$$
\rho_{2}(n)=p^{-1} \circ \rho_{1}(n) \circ p \quad \forall n \in N .
$$

\section{Groups of AFFine DefeCt OnE}

The only known connected, simply connected nilpotent Lie groups not acting simply transitively and affinely on Euclidean $n$-space are those constructed by Benoist [Ben92] and Burde and Grunewald [BG93]. In fact, it is the situation on the Lie algebra level which is investigated in both papers.

The Lie algebras in question are 11-dimensional; a particularly interesting family of them is denoted by $\mathfrak{a}(-2, s, t)$ and has a basis $e_{1}, e_{2}, \ldots, e_{11}$. Moreover, the brackets of these Lie algebras are completely determined by

$$
\begin{aligned}
& {\left[e_{1}, e_{i}\right]=e_{i+1}, \quad 2 \leq i \leq 10,} \\
& {\left[e_{1}, e_{11}\right]=0} \\
& {\left[e_{2}, e_{3}\right]=e_{5} \text { and }} \\
& {\left[e_{2}, e_{5}\right]=-2 e_{7}+s e_{8}+t e_{9} .}
\end{aligned}
$$

The results of Benoist, Burde and Grunewald imply that, in case $s \neq 0, \mathfrak{a}(-2, s, t)$ has no faithful 12-dimensional linear representation. Therefore, no lattice of the corresponding Lie group $\mathcal{A}(-2, s, t)$ can act properly discontinuously on $\mathbb{R}^{11}$, via affine motions and with compact quotient.

In this section we indicate how one can construct a uniform lattice $\Gamma$ of $\mathcal{A}(-2, s, t)$, acting properly discontinuously on $\mathbb{R}^{11}$ via polynomial diffeomorphisms, in such a way that the polynomial mappings used are of degree $\leq 2$. It will follow that these lattices $\Gamma$ have affine defect 1 .

To do so, we will embed this $\Gamma$ into a connected, simply connected Lie group $P$ (of finite dimension) of polynomial diffeomorphisms of $\mathbb{R}^{11}$. In fact, let $P$ be the 
group consisting of all mappings

$$
f: \mathbb{R}^{11} \rightarrow \mathbb{R}^{11}:\left(\begin{array}{c}
y_{11} \\
y_{10} \\
\vdots \\
y_{2} \\
y_{1}
\end{array}\right) \mapsto\left(\begin{array}{c}
y_{11}+p_{11}\left(y_{1}, y_{2}, \ldots, y_{10}\right) \\
y_{10}+p_{10}\left(y_{1}, y_{2}, \ldots, y_{9}\right) \\
\vdots \\
y_{2}+p_{2}\left(y_{1}\right) \\
y_{1}+p_{1}
\end{array}\right)
$$

where $p_{i}\left(y_{1}, y_{2}, \ldots, y_{i-1}\right)$ is a polynomial of degree $\leq 1$ in the variables $y_{1}, \ldots, y_{i-1}$, except $p_{11}$ which is of degree $\leq 2$.

In view of the results mentioned above, an embedding $\Gamma \hookrightarrow P$ is really the best (non affine) thing we could expect.

The first step to clear this job was to construct a unitriangular matrix representation of $\mathcal{A}(-2, s, t)$. This was not difficult since Burde and Grunewald [BG93] give an explicit way to construct a 34-dimensional (and with some more trouble, a 22-dimensional) matrix representation for the Lie algebra $\mathfrak{a}(-2, s, t)$, using its universal enveloping algebra. These matrices are upper triangular, with 0's on the diagonal, and so by exponentiating we find the desired representation on the Lie group level.

For the sake of simplicity we will restrict ourselves to the cases where $s, t \in \mathbb{Z}$; however, we remark here that things work out for rational $s$ and $t$ too.

Let $\Gamma$ denote the subgroup of $\mathcal{A}(-2, s, t)$ generated by $A_{1}, A_{2}, \ldots, A_{11}$, where

$$
\begin{array}{lll}
A_{1}=\exp \left(e_{1}\right) & A_{2}=\exp \left(e_{2}\right) & A_{3}=\exp \left(e_{3}\right) \\
A_{4}=\exp \left(\frac{1}{2} e_{4}\right) & A_{5}=\exp \left(\frac{1}{6} e_{5}\right) & A_{6}=\exp \left(\frac{1}{24} e_{6}\right) \\
A_{7}=\exp \left(\frac{1}{120} e_{7}\right) & A_{8}=\exp \left(\frac{1}{720} e_{8}\right) & A_{9}=\exp \left(\frac{1}{25200} e_{9}\right) \\
A_{10}=\exp \left(\frac{1}{1008000} e_{10}\right) & A_{11}=\exp \left(\frac{1}{90720000} e_{11}\right) &
\end{array} .
$$

Making use of the matrix representation of $\mathcal{A}(-2, s, t)$, and so of $\Gamma$, mentioned above, one checks that $\Gamma$ is a uniform lattice of $\mathcal{A}(-2, s, t)$ with

$$
\begin{aligned}
& \Gamma=\left\langle A_{1}, \ldots, A_{11} \|\left[A_{2}, A_{1}\right]=A_{3}^{-1} A_{4} A_{5}^{2} A_{6}^{-5} A_{7}^{109} A_{8}^{4(79-60 s)} A_{9}^{6(-1108-525 s-1400 t)}\right. \\
& \text {. } A_{10}^{-2267555+151088 s-126000 t} \\
& \text {. } A_{11}^{2\left(203230225-12930435 s+677376 s^{2}+4372200 t\right)} \\
& {\left[A_{3}, A_{1}\right]=A_{4}^{-2} A_{5}^{3} A_{6}^{-4} A_{7}^{185} A_{8}^{6(-91-60 s)} A_{9}^{35(829+180 s-360 t)}} \\
& \text {. } A_{10}^{80}(45515-3066 s+3150 t) \\
& \text {. } A_{11}^{180\left(-5202055+135870 s+18816 s^{2}-190050 t\right)} \\
& {\left[A_{3}, A_{2}\right]=A_{5}^{-6} A_{7}^{-120} A_{8}^{360(3+s)} A_{9}^{840(-26-15 s+15 t)}} \\
& \text {. } A_{10}^{6720(-585-28 s-75 t)} A_{11}^{3780\left(-41975-13035 s-2688 s^{2}+5150 t\right)} \\
& {\left[A_{4}, A_{1}\right]=A_{5}^{-3} A_{6}^{6} A_{7}^{-10} A_{8}^{15} A_{9}^{16905} A_{10}^{140(-2425-864 s)}} \\
& \text {. } A_{11}^{90\left(836225+60480 s+28224 s^{2}-222075 t\right)} \text {, } \\
& {\left[A_{4}, A_{2}\right]=A_{6}^{-12} A_{8}^{-900} A_{9}^{12600 s} A_{10}^{8400(341+60 t)} A_{11}^{13494600 s} \text {, }} \\
& {\left[A_{4}, A_{3}\right]=A_{7}^{-180} A_{8}^{360 s} A_{9}^{12600 t} A_{10}^{-5896800} A_{11}^{-85050(4725+334 s)} \text {, }} \\
& {\left[A_{5}, A_{1}\right]=A_{6}^{-4} A_{7}^{10} A_{8}^{-20} A_{9}^{175} A_{10}^{-1400} A_{11}^{21708750} \text {, }} \\
& {\left[A_{5}, A_{2}\right]=A_{7}^{40} A_{8}^{-120 s} A_{9}^{840(13-5 t)} A_{10}^{94080 s} A_{11}^{1260\left(-12350+4032 s^{2}-7725 t\right)} \text {, }}
\end{aligned}
$$




$$
\begin{aligned}
& \Gamma \text {-continued }: \quad\left[A_{5}, A_{3}\right]=A_{8}^{-360} A_{9}^{4200 s} A_{10}^{168000 t} A_{11}^{91003500}, \\
& {\left[A_{5}, A_{4}\right]=A_{9}^{-11340} A_{10}^{80640 s} A_{11}^{3780\left(-448 s^{2}+3525 t\right)} \text {, }} \\
& {\left[A_{6}, A_{1}\right]=A_{7}^{-5} A_{8}^{15} A_{9}^{-175} A_{10}^{1750} A_{11}^{-31500},} \\
& {\left[A_{6}, A_{2}\right]=A_{8}^{150} A_{9}^{-2100 s} A_{10}^{42000(-13-2 t)} A_{11}^{-6095250 s} \text {, }} \\
& {\left[A_{6}, A_{3}\right]=A_{9}^{2520} A_{10}^{1680 s} A_{11}^{1890\left(448 s^{2}-1525 t\right)} \text {, }} \\
& {\left[A_{6}, A_{4}\right]=A_{10}^{-113400} A_{11}^{1814400 s},} \\
& {\left[A_{6}, A_{5}\right]=A_{11}^{-10843875}} \\
& {\left[A_{7}, A_{1}\right]=A_{8}^{-6} A_{9}^{105} A_{10}^{-1400} A_{11}^{31500},} \\
& {\left[A_{7}, A_{2}\right]=A_{9}^{546} A_{10}^{-17136 s} A_{11}^{189\left(-6175-896 s^{2}-4950 t\right)} \text {, }} \\
& {\left[A_{7}, A_{3}\right]=A_{10}^{65520} A_{11}^{-695520 s} \text {, }} \\
& {\left[A_{7}, A_{4}\right]=A_{11}^{4465125} \text {, }} \\
& {\left[A_{8}, A_{1}\right]=A_{9}^{-35} A_{10}^{700} A_{11}^{-21000},} \\
& {\left[A_{8}, A_{2}\right]=A_{10}^{-7280} A_{11}^{-141120 s} \text {, }} \\
& {\left[A_{8}, A_{3}\right]=A_{11}^{-505575} \text {, }} \\
& {\left[A_{9}, A_{1}\right]=A_{10}^{-40} A_{11}^{1800} \text {, }} \\
& {\left[A_{9}, A_{2}\right]=A_{11}^{-4275} \text {, }} \\
& {\left[A_{10}, A_{1}\right]=A_{11}^{-90} \text {, }} \\
& \left.\left[A_{i}, A_{j}\right]=0 \text { for } 1 \leq j<i \leq 11 \text { and } i+j>11\right\rangle \text {. }
\end{aligned}
$$

In fact, after having taken $A_{1}=\exp \left(e_{1}\right)$ and $A_{2}=\exp \left(e_{2}\right)$, the others have been chosen carefully so that they generate a lattice. It is obvious that this choice is not unique.

Now that we have enough information about the group structure, we are going to build up an embedding $\Gamma \rightarrow P$. In order to do so we will first realise the rank 10 group $\Gamma / Z(\Gamma)=\Gamma /\left\langle A_{11}\right\rangle$ as a properly discontinuous group of affine motions of $\mathbb{R}^{10}$. The idea behind this is the fact that the adjoint representation ad $: \mathfrak{a}(-2, s, t) \rightarrow \mathfrak{g l}(\mathfrak{a}(-2, s, t))$ is an 11-dimensional matrix representation factoring through $\mathfrak{a}(-2, s, t) / Z(\mathfrak{a}(-2, s, t))=\mathfrak{a}(-2, s, t) /\left\langle e_{11}\right\rangle$. By exponentiating this representation, we may realise $\mathcal{A}(-2, s, t) / Z(\mathcal{A}(-2, s, t))$ as a group of affine motions of $\mathbb{R}^{10}$. However, in this way the group $\mathcal{A}(-2, s, t) / Z(\mathcal{A}(-2, s, t))$ does not act simply transitively on $\mathbb{R}^{10}$ as we would like, therefore we slightly modify the adjoint representation in order to obtain, on the Lie group level, a simply transitive action. This altered adjoint representation, denoted by $\phi$, is determined completely by the images of $e_{1}$ and $e_{2}$ :

$$
\phi\left(e_{1}\right)=\left(\begin{array}{ccccccccccc}
0 & 1 & 0 & 0 & 0 & 0 & 0 & 0 & 0 & 0 & 0 \\
0 & 0 & 1 & 0 & 0 & 0 & 0 & 0 & 0 & 0 & 0 \\
0 & 0 & 0 & 1 & 0 & 0 & 0 & 0 & 0 & 0 & 0 \\
0 & 0 & 0 & 0 & 1 & 0 & 0 & 0 & 0 & 0 & 0 \\
0 & 0 & 0 & 0 & 0 & 1 & 0 & 0 & 0 & 0 & 0 \\
0 & 0 & 0 & 0 & 0 & 0 & 1 & 0 & 0 & 0 & 0 \\
0 & 0 & 0 & 0 & 0 & 0 & 0 & 1 & 0 & 0 & 0 \\
0 & 0 & 0 & 0 & 0 & 0 & 0 & 0 & 1 & 0 & 0 \\
0 & 0 & 0 & 0 & 0 & 0 & 0 & 0 & 0 & 0 & 0 \\
0 & 0 & 0 & 0 & 0 & 0 & 0 & 0 & 0 & 0 & -1 \\
0 & 0 & 0 & 0 & 0 & 0 & 0 & 0 & 0 & 0 & 0
\end{array}\right)
$$


and

$$
\phi\left(e_{2}\right)=\left(\begin{array}{ccccccccccc}
0 & 0 & \frac{19}{16} & \frac{28 s}{25} & \frac{448 s^{2}+2475 t}{2000} & 0 & 0 & 0 & 0 & 0 & 0 \\
0 & 0 & 0 & \frac{26}{5} & \frac{51 s}{25} & 2 t & 0 & 0 & 0 & 0 & 0 \\
0 & 0 & 0 & 0 & -\frac{13}{5} & 2 s & t & 0 & 0 & 0 & 0 \\
0 & 0 & 0 & 0 & 0 & -5 & s & 0 & 0 & 0 & 0 \\
0 & 0 & 0 & 0 & 0 & 0 & -2 & 0 & 0 & 0 & 0 \\
0 & 0 & 0 & 0 & 0 & 0 & 0 & 1 & 0 & 0 & 0 \\
0 & 0 & 0 & 0 & 0 & 0 & 0 & 0 & 1 & 0 & 0 \\
0 & 0 & 0 & 0 & 0 & 0 & 0 & 0 & 0 & 0 & 0 \\
0 & 0 & 0 & 0 & 0 & 0 & 0 & 0 & 0 & 0 & -1 \\
0 & 0 & 0 & 0 & 0 & 0 & 0 & 0 & 0 & 0 & 0 \\
0 & 0 & 0 & 0 & 0 & 0 & 0 & 0 & 0 & 0 & 0
\end{array}\right) .
$$

Let us define the morphism $\varphi$ as the composite map:

$$
\mathcal{A}(-2, s, t) \stackrel{\log }{\longrightarrow} \mathfrak{a}(-2, s, t) \longrightarrow \mathfrak{a}(-2, s, t) /\left\langle e_{11}\right\rangle \stackrel{\phi}{\longrightarrow} \mathfrak{g l}(11, \mathbb{R}) \stackrel{\exp }{\longrightarrow} G l(11, \mathbb{R})
$$

As the images of $\varphi$ consist of unitriangular matrices, $\mathcal{A}(-2, s, t) / Z(\mathcal{A}(-2, s, t))$ can indeed be looked at as being a simply transitive group of affine motions of $\mathbb{R}^{10}$.

In the next step we lift the map $\varphi: \Gamma \rightarrow G l(11, \mathbb{R})$ to a faithful morphism $\psi: \Gamma \rightarrow P$. We present $\psi$ by giving the images of the generators:

$$
\psi\left(A_{i}\right): \mathbb{R}^{11} \rightarrow \mathbb{R}^{11}:\left(\begin{array}{c}
y_{11} \\
y_{10} \\
\vdots \\
y_{2} \\
y_{1}
\end{array}\right) \mapsto\left(\begin{array}{c}
y_{11}+f_{i}\left(y_{1}, y_{2}, \ldots, y_{10}\right) \\
\varphi\left(A_{i}\right)\left(\begin{array}{c}
y_{10} \\
\vdots \\
y_{2} \\
y_{1}
\end{array}\right)
\end{array}\right)
$$

Here, we see $\varphi\left(A_{i}\right)$ as an affine mapping of $\mathbb{R}^{10}$ and the $f_{i}$ are polynomials of degree $\leq 2$ given by:

$$
\begin{aligned}
f_{1}\left(y_{1}, \ldots, y_{10}\right)= & \frac{99569925 t+17013024 s^{2}-250}{90720000} y_{2}-\frac{1}{40320} y_{3}-\frac{1}{5040} y_{4} \\
& -\frac{1}{720} y_{5}-\frac{1}{120} y_{6}-\frac{1}{24} y_{7}-\frac{1}{6} y_{8}-\frac{1}{2} y_{9}-y_{10} \\
& +\frac{-5458475+7392820 s+1937376 s^{2}+10623200 t}{1680000} y_{2} y_{3} \\
& +\frac{-142755-6860 s+3136 s^{2}-38675 t}{84000} y_{2} y_{4} \\
& +\frac{1617-320 s-960 t}{1920} y_{2} y_{5}+\frac{-800-40 s-448 s^{2}+1525 t}{2000} y_{2} y_{6} \\
& +\frac{-195+46 s}{50} y_{2} y_{7}+\frac{321}{80} y_{2} y_{8} \\
& +\frac{38025-2560 s+1792 s^{2}-14100 t}{16000} y_{3} y_{4}
\end{aligned}
$$


POLYNOMIAL STRUCTURES FOR NILPOTENT GROUPS

$$
\begin{aligned}
& +\frac{15075-1920 s+896 s^{2}-7050 t}{4000} y_{3} y_{5}+\frac{3(45-16 s)}{50} y_{3} y_{6} \\
& -\frac{189}{16} y_{3} y_{7}+\frac{1377}{160} y_{4} y_{5}+\frac{1377}{80} y_{4} y_{6} \\
& -\frac{6486150-22287100 s-5727456 s^{2}-1128960 s^{3}-32661825 t}{10080000} y_{2}^{2} \\
& +\frac{11881800 s t}{10080000} y_{2}^{2}+\frac{-627615-13440 s+12544 s^{2}-98700 t}{336000} y_{3}^{2} \\
& +\frac{459}{160} y_{4}^{2} \\
& f_{2}\left(y_{1}, \ldots, y_{10}\right)=\frac{-69500-3263904 s^{2}-376320 s^{3}+6044325 t-3960600 s t}{3360000} y_{3} \\
& +\frac{21465 s-6272 s^{3}-66010 s t}{28000} y_{4} \\
& +\frac{-8843500-2685984 s^{2}+7797825 t}{1680000} y_{5}+\frac{198 s}{875} y_{6} \\
& +\frac{15775-6272 s^{2}-34650 t}{28000} y_{7}+\frac{-28 s}{25} y_{8}+\frac{-19}{16} y_{9} \\
& +\frac{-767800+210464 s^{2}-120825 t}{160000} y_{2} y_{3} \\
& +\frac{-433649 s+6272 s^{3}+66010 s t}{28000} y_{2} y_{4} \\
& +\frac{519000+83104 s^{2}-218825 t}{40000} y_{2} y_{5}+\frac{517 s}{14000} y_{2} y_{6}-\frac{4089}{560} y_{2} y_{7} \\
& +\frac{-11391300+309792 s^{2}+2217775 t}{560000} y_{3} y_{4}+\frac{1671 s}{350} y_{3} y_{5} \\
& +\frac{21087}{1400} y_{3} y_{6}-\frac{5913}{560} y_{4} y_{5} \\
& +\frac{-16554375-339872 s+150528 s^{3}+1584240 s t}{1344000} y_{2}^{2} \\
& +\frac{1671 s}{700} y_{3}^{2}+\frac{-33039 s}{2800} y_{4}^{2} \\
& f_{3}\left(y_{1}, \ldots, y_{10}\right)=\frac{3(2187-3584 s)}{2800} y_{4}-\frac{62901}{2800} y_{5}+\frac{448 s^{2}-1525 t}{2000} y_{6}+\frac{-23 s}{25} y_{7} \\
& -\frac{321}{80} y_{8}+\frac{-41175-6272 s^{3}-66010 s t}{28000} y_{2} y_{3} \\
& +\frac{11\left(-9184 s^{2}+29075 t\right)}{80000} y_{2} y_{4}+\frac{-19407 s}{14000} y_{2} y_{5}-\frac{549}{560} y_{2} y_{6} \\
& +\frac{3573 s}{400} y_{3} y_{4}+\frac{76383}{1400} y_{3} y_{5}+\frac{-26336 s^{2}-457825 t}{80000} y_{3}^{2}-\frac{2673}{112} y_{4}^{2}, \\
& f_{4}\left(y_{1}, \ldots, y_{10}\right)=\frac{-448 s^{2}+3525 t}{4000} y_{5}+\frac{12 s}{25} y_{6}+\frac{189}{32} y_{7}+\frac{26336 s^{2}+457825 t}{160000} y_{2} y_{3} \\
& -\frac{123183 s}{28000} y_{2} y_{4}-\frac{35397}{1120} y_{2} y_{5}+\frac{6561}{1400} y_{3} y_{4}+\frac{448 s^{3}+4715 s t}{4000} y_{2}^{2},
\end{aligned}
$$




$$
\begin{aligned}
f_{5}\left(y_{1}, \ldots, y_{10}\right)= & \frac{3157 s^{2}}{15000} y_{2}^{2}-\frac{12793 t}{19200} y_{2}^{2}-\frac{39 s}{1750} y_{2} y_{3} \\
& -\frac{2187}{1400} y_{3}^{2}+\frac{891}{224} y_{2} y_{4}-\frac{459}{160} y_{6}, \\
f_{6}\left(y_{1}, \ldots, y_{10}\right)= & \frac{6469 s}{112000} y_{2}^{2}+\frac{8073}{22400} y_{2} y_{3}, \\
f_{7}\left(y_{1}, \ldots, y_{10}\right)= & \frac{183}{22400} y_{2}^{2} \\
f_{8}\left(y_{1}, \ldots, y_{10}\right)= & 0 \\
f_{9}\left(y_{1}, \ldots, y_{10}\right)= & 0 \\
f_{10}\left(y_{1}, \ldots, y_{10}\right)= & 0 \\
f_{11}\left(y_{1}, \ldots, y_{10}\right)= & \frac{1}{90720000} .
\end{aligned}
$$

Theorem 5.1. Let $\mathcal{A}(-2, s, t)$ be the Lie group which stands in one-to-one correspondence with the Lie algebra $\mathfrak{a}(-2, s, t)$. If $s, z \in \mathbb{Z}$, then $\mathcal{A}(-2, s, t)$ acts simply transitively and via polynomial diffeomorphisms of degree $\leq 2$ on $\mathbb{R}^{11}$. So, if $s \neq 0$, then any lattice of $\mathcal{A}(-2, s, t)$ is a group of affine defect one.

Proof. By the computations above we know that $\mathcal{A}(-2, s, t)$ contains a lattice $\Gamma$ which embeds nicely into a Lie group $P$ of polynomial diffeomorphisms of degree $\leq 2$. Observe that this group $P$, introduced above, is nilpotent. This can be seen as follows. Let $P_{1}$ be the normal subgroup of $P$ consisting of those polynomial diffeomorphisms for which $p_{i} \equiv 0(1 \leq i \leq 10)$. So $P_{1}$ consists of those polynomials of $\mathbb{R}^{11}$ which only affect 1 coordinate. Denote by $P_{2}$ the subgroup of $P$ determined by those polynomials for which $p_{11} \equiv 0$. So $P_{2}$ is isomorphic to the group of unitriangular $11 \times 11-$ matrices. There is a split short exact sequence

$$
1 \rightarrow P_{1} \rightarrow P \rightarrow P_{2} \rightarrow 1
$$

$P_{2}$ is a nilpotent group, and so $\gamma_{n}(P) \subseteq P_{1}$ if $n$ is big enough. It is now an exercise to see that further commutator subgroups will gradually get smaller and smaller until, at the end, they vanish.

Using [Gor71, Proposition 2.5], the embedding $\Gamma \rightarrow P$ extends uniquely to an embedding of the Lie group $\mathcal{A}(-2, s, t) \rightarrow P$, making it acting properly discontinuously on $\mathbb{R}^{11}$, which was to be shown.

Let us point out finally that the use of MATHEMATICA ${ }^{\circledR}$ [Wol93] was quite crucial in setting up and performing the computations above. A set of procedures enabling the reader to verify these results is available on request from the authors.

\section{REFERENCES}

[Ben92] Yves Benoist, Une nilvariété non affine, C. R. Acad. Sci. Paris Sér. I Math. 315 (1992), pp. 983-986. MR 93j:22008

[Ben95] Yves Benoist, Une nilvariété non affine, J. Differential Geom. 41 (1995), pp. 21-52.

[BG93] Dietrich Burde and Fritz Grunewald, Modules for certain lie algebras of maximal class, Preprint (1993), to appear in J. Pure and Applied Algebra.

[Boy89] Nguiffo B. Boyom, The lifting problem for affine structures in nilpotent lie groups, Trans. Amer. Math. Soc. (1989), pp. 347-379. MR 89i:53035

[Boy90] Nguiffo B. Boyom, Sur les structures affines homotopes à zèro des groupes de lie, J. Differential Geom. (1990), pp. 859-911. MR 91g:53026 
[CR77] Pierre E. Conner and Frank Raymond, Deforming homotopy equivalences to homeomorphisms in aspherical manifolds, Bull. A.M.S. 83 (1977), pp. 36-85. MR 57:7629

[DI93] Karel Dekimpe and Paul Igodt, Computational aspects of affine representations for torsion free nilpotent groups via the seifert construction, J. Pure Applied Algebra (1993), pp. 165-190. MR 94e:20049

[DI94] Karel Dekimpe and Paul Igodt, The structure and topological meaning of almost-torsion free groups, Comm. Algebra 22 (1994), pp. 2547-2558. MR 95f:20057

[FG83] David Fried and William M. Goldman, Three-dimensional affine crystallographic groups, Adv. in Math. 47 (1983), pp. 1-49. MR 84d:20047

[Gor71] V. V. Gorbacevič, Discrete subgroups of solvable Lie groups of type (E), Math. USSR Sbornik 14 (1971), pp. 233-251. MR 36:6448

[Har91] Manfred Hartl, Abelsche Modelle Nilpotenter Gruppen, Inaugural-Dissertation RFW Univ. Bonn, 1991.

[IL90] Paul Igodt and Kyung Bai Lee, On the uniqueness of certain affinely flat infranilmanifolds, Math. Zeitschrift 204 (1990), pp. 605-613. MR 91e:57066

[Lee83] Kyung Bai Lee, Aspherical manifolds with virtually 3-step nilpotent fundamental group, Amer. J. Math. 105 (1983), pp. 1435-1453. MR 85d:57032

[LR81] Kyung Bai Lee and Frank Raymond, Topological, affine and isometric actions on flat riemannian manifolds, J. Differential Geometry 16 (1981), pp. 255-269. MR 84k:57027

[LR84] Kyung Bai Lee and Frank Raymond, Geometric realization of group extensions by the seifert construction, Contemporary Math. A. M. S. 33 (1984), pp. 353-411. MR 86h:57043

[Mal51] Anatoliǐ I. Mal'cev, On a class of homogeneous spaces, Translations A.M.S. (1951), no. 39, pp. 1-33. MR 12:589e

[Mil77] John Milnor, On fundamental groups of complete affinely flat manifolds, Adv. Math. 25 (1977), pp. 178-187. MR 56:13130

[Nis90] Mounir Nisse, Structure affine des infranilvariétés et infrasolvariétés, C. R. Acad. Sci. Paris Série I 310 (1990), pp. 667-670. MR 91e:57069

[Sch74] John Scheuneman, Affine structures on three-step nilpotent lie algebras, Proc. A.M.S. 46 (1974), pp. 451-454. MR 54:470

[Seg83] Daniel Segal, Polycyclic Groups, Cambridge University Press, 1983. MR 85h:20003

[Wol93] Stephen Wolfram, Mathematica, Wolfram Research, Inc., 1993.

Katholieke Universiteit Leuven, Campus Kortrijk, B-8500 Kortrijk, Belgium

E-mail address: Paul.Igodt@kulak.ac.be

Department of Mathematics, University of Oklahoma, Norman, Oklahoma 73019

E-mail address: kblee@dstn3.math.uoknor.edu 